\title{
A Logic Approach for LTL System Modification
}

\author{
Yulin Ding and Yan Zhang \\ School of Computing \& Information Technology \\ University of Western Sydney \\ Kingswood, N.S.W. 1797, Australia \\ email: \{yding,yan\}@cit.uws.edu.au
}

\begin{abstract}
Model checking has been successfully applied to system verification. However, there are no standard and universal tools to date being applied for system modification. This paper introduces a formal approach called the Linear Temporal Logic (LTL) model update for system modification. In contrast to previous error repairing methods, which were usually simple program debugging and specialized technical methods, our LTL model update modifies the existing LTL model of an abstracted system to correct automatically the errors occurring within this model. We introduce three single operations to represent, update, and simplify the updating problem. The minimal change rules are then defined based on such update operations. We show how our approach can eventually be applied in system modifications by illustrating an example of program corrections and characterizing some frequently used properties in the LTL Kripke model.
\end{abstract}

Key words: Logic for Artificial Intelligence, belief revision and update, temporal reasoning, model update, model checking

\section{Introduction}

Model checking is rather mature in both theoretical and practical research. Currently, model checkers with SMV or Promela [7] series as their specification languages are widely available for research, experiment, and partial industry purposes. Nowadays SMV [3], NuSMV, Cadence SMV [9] and SPIN [7] have been well accepted as the state of the art model checkers. In most model checkers, counterexamples are the major mechanisms to report errors. However, in Cadence SMV, the counterexample-free concept is introduced [9].

Model checking is a tool to report errors of a designed system. The next step is to repair the errors. In 1998, Stumptner and Wotawa presented an overview of Artificial Intelligence approaches to the development of intelligent debugging systems in [10]. However, most of the previous methods concerning system repair were localized or detailed methods oriented. Recently, error diagnosis and repair are starting to employ a formal methods approach. Buccafurri et. al. [2] applied Artificial Intelligence techniques to model checking and error repairing. They 
used abductive model revision techniques, repairing errors in concurrent programs. It is a new approach towards automated diagnosis and integrated repair on model checking. It aims at using techniques and concepts from model checking by combining them with AI principles. Harris and Ryan [5] recently proposed another attempt of system modification, which designed update operations to tackle feature integration performing theory change and belief revision.

The update of the knowledge base has been extensively researched. Winslett [12] was a pioneer of the update of the knowledge base and used series update and minimal change methods for databases. Hezig and Rifi [6] conducted extensive surveys about updates of the knowledge base and their development before 1999. They listed ten update operations, based on propositional logic and further characteristics. Recently, the update of the knowledge base is enhanced by the modality update by Baral and Zhang [1]. They discussed knowledge update and its minimal change, based on modal logic $S 5$. Both the update of the knowledge base and the knowledge update are at the stage of theoretical research.

We intend to apply knowledge update to practice and develop a universal method to repair errors automatically as the main contribution of our research. In particular, we propose a general method of the Linear Temporal Logic (LTL) model update, by integrating the ideas of knowledge update and model checking in this paper.

This paper is organized as follows: Section 2 reviews LTL syntax and semantics, with the state of the art LTL model checking. Section 3 introduces two major definitions for model update. Section 4 introduces the minimal change principles of model update. Section 5 characterizes two important properties in the LTL model for its update. Finally, the paper concludes with Section 6 .

\section{LTL Kripke Model Checking}

\subsection{Knowledge about LTL}

Definition 1. [3] Let AP be a set of atomic propositions. A Kripke model $M$ over $A P$ is a four tuple $M=\left(S, S_{0}, R, L\right)$ where

1. $S$ is a finite set of states.

2. $S_{0} \subseteq S$ is the set of initial states.

3. $R \subseteq S \times S$ is a transition relation.

4. $L: S \rightarrow 2^{A P}$ is a function that assigns each state with a set of atomic propositions.

Definition 2. [8] Linear-time temporal logic (LTL) has the following syntax given in Backus naur form:

$$
\phi::=p|(\neg \phi)|(\phi \wedge \phi)|(\phi \cup \phi)|(G \phi)|(F \phi)|(X \phi)
$$

where $p$ is any propositional atom. 
An LTL formula is evaluated on a path, a sequence of states, or a set of paths. Consider the path $\pi \stackrel{\text { def }}{=}\left[s_{0}, s_{1}, s_{2}, \cdots\right]$; we write $\pi^{i}$ for the suffix starting at $s_{i}$, i.e. $\pi^{i}$ is $\left[s_{i}, s_{i+1}, \cdots\right]$, and $\pi^{[i]}$ for the prefix ending at $s_{i}$, i.e. $\pi^{[i]}=\left[s_{0}, \cdots, s_{i-1}, s_{i}\right]$. We write $\left(s_{i}, s_{i+1}\right) \subseteq \pi$ and $s_{i} \in \pi$, if $\pi=\left[s_{0}, \cdots, s_{i}, s_{i+1}, \cdots\right]$.

Definition 3. [8] Let $M=\left(S, S_{0}, R, L\right)$ be a Kripke model. Then a path of $M$ is $\pi=\left[s_{0}, s_{1}, s_{2}, \cdots\right]$, where for all $i=0,1, \cdots$, we have $s_{0} \in S_{0}$, and $\left(s_{i}, s_{i+1}\right) \in R$. $W e$ define when $\pi$ satisfies an LTL formula via the satisfaction relation $\models$ for LTL formulas as follows:

1. $\pi \models \top$.

2. $\pi \models p$ iff $p \in L\left(s_{0}\right)$.

3. $\pi \models \neg \phi$ iff $\pi \not \models \phi$.

4. $\pi \models \phi_{1} \wedge \phi_{2}$ iff $\pi \models \phi_{1}$ and $\pi \models \phi_{2}$.

5. $\pi \models X \phi$ iff $\pi^{1} \models \phi$.

6. $\pi \models G \phi$ holds iff, for all $i \geq 0, \pi^{i} \models \phi$.

7. $\pi \models F \phi$ holds iff, for some $i \geq 0, \pi^{i} \models \phi$.

8. $\pi=\phi \cup \psi$ holds iff there is some $i \geq 0$ such that $\pi^{i} \models \psi$ and for all $j=1, \ldots, i-1$ we have $\pi^{j}=\phi$.

We say $M$ satisfies $\phi$, denoted as $M \models \phi$, if for each path $\pi$ of $M$,starting from an initial state, $\pi \models \phi$.

Although the syntax of LTL is thought of as only one path at a time, the semantic viewpoint of LTL is that all paths are considered [8]. In this paper, all the update operations are executed one path at a time, from the syntax viewpoint of LTL. The update and the minimal change methods only apply to finite paths. The LTL representations of software systems in examples within this paper are done by identical methods as the first software system represented in CTL in [11]. That is, one state represents one changed value of the variable in the running process of a software system.

\subsection{LTL Model Checking: An Overview}

In the context of model checking, a designed system is represented by the CTL or LTL Kripke structure $M$. Then $M$ should satisfy a certain property $\phi$. The function of a model checker is to check whether $M$ really satisfies $\phi$. If it does not, the counterexamples [3] report the errors. The describing languages to perform the model checking are, for example, SMV for LTL and CTL, and Promela for LTL.

Parallel to CTL model checking, LTL model checking is extensively studied. One of the main streams is to perform LTL model checking through SMV. In the early stages, Clarke et al. in [3] translated LTL to CTL model checking with fairness constraints, adding a translator into SMV. In recent years, new model checkers such as NuSMV, Cadence SMV and MCK [4] use similar functions of Clarke's LTL-to-CTL translation. Another main stream for LTL model checking is to translate LTL into automata, such as the current popular LTL model 
checker SPIN. For SPIN, Promela is its specification language in contrast to SMV. SPIN supports the design and verification of asynchronous process software systems. It performs on-the-fly model checking. We have investigated that LTL model checking is well developed for research purposes and perceived as a potential industrial technique. However, model update based on LTL systems has not been explored by researchers yet. We address in the following sections detailed ideas and methods for LTL model updating.

\section{The LTL Kripke Model Update: Formal Definitions}

Model update is a new concept for system verification and modification. If a designed system $M$ does not satisfy a property $\phi$ after model checking(See Fig. 1.), the model updater automatically corrects errors in $M$, updating $M$ to the new model $M^{\prime}$. Thus, $M^{\prime} \models \phi$. Model update is implemented by the model updater. Now we define three update operations for LTL model update on the basis of state of an LTL Kripke model, which is a Kripke model with syntaxes in Defintion 2 and semantics in Definition 3.

\begin{tabular}{|c|c|c|c|c|}
\hline M & A model & M & $\sqrt{\text { A model }}$ & M' \\
\hline$\phi ?$ & checker & $-\phi$ & updater & $\phi$ \\
\hline
\end{tabular}

Fig. 1. The relationship of a model checker and a model updater.

Definition 4. (Simple Modification) Given an LTL Kripke structure, $M=$ $\left(S, S_{0}, R, L\right)$ over $A P$, where $S_{0} \subseteq S$. A simple modification on $M$ is defined as one of the following three single operations: ${ }^{1}$

-State Addition: $\operatorname{add}\left(M, s_{\text {new }}, s_{k}, s_{k+1}\right)=M^{\prime}=\left(S^{\prime}, S_{0}^{\prime}, R^{\prime}, L^{\prime}\right)$ over $A P$, where

$S^{\prime}=S \cup\left\{s_{\text {new }}\right\} ;$

$S^{\prime}{ }_{0}=\left\{\begin{array}{l}S_{0} \cup\left\{s_{\text {new }}\right\}-\left\{s_{k+1}\right\}, \quad \text { if } s_{k+1} \in S_{0} \text { and } s_{\text {new }} \in S_{0}^{\prime} \\ S_{0}, \quad \text { otherwise; }\end{array}\right.$

$R^{\prime}=\left\{\begin{array}{l}R \cup\left\{\left(s_{\text {new }}, s_{k+1}\right)\right\}, \text { if } s_{k+1} \in S_{0} ; \\ R \cup\left\{\left(s_{k}, s_{\text {new }}\right)\right\}, \text { if }\left(s_{k}, s_{k+1}\right) \notin R ; \\ R \cup\left\{\left(s_{k}, s_{\text {new }}\right),\left(s_{\text {new }}, s_{k+1}\right)\right\}-\left\{\left(s_{k}, s_{k+1}\right)\right\}, \text { otherwise }\end{array}\right.$

$L^{\prime}: S^{\prime} \rightarrow 2^{A P}$, where $\forall s \in S^{\prime}$, if $s \in S$, then $L^{\prime}(s)=L(s)$, else

$L^{\prime}\left(s_{\text {new }}\right)=\tau\left(s_{\text {new }}\right)$, where $\tau$ is the truth assignment related to $s_{\text {new }}$.

-State Deletion: delete $\left(M, s_{k}\right)=M^{\prime}=\left(S^{\prime}, S_{0}^{\prime}, R^{\prime}, L^{\prime}\right)$ over AP, where $S^{\prime}=S-\left\{s_{k}\right\}$

\footnotetext{
${ }^{1}$ In the following statement, $s_{k}$ and $s_{k+1}$ are refered to the $k$-th and $k+1$-th states of a path in $M$. If $s_{k}$ is the last state of a path, $s_{k+1}$ does not exist.
} 
$S^{\prime}{ }_{0}=\left\{\begin{array}{l}S_{0}-\left\{s_{k}\right\}, \text { if } s_{k} \in S_{0} \\ S_{0}, \text { otherwise }\end{array}\right.$

$R^{\prime}=\left\{\begin{array}{l}R-\left\{\left(s_{k}, s_{k+1}\right)\right\}, \text { if } s_{k} \in S_{0} \text { and }\left(s_{k}, s_{k+1}\right) \in R \\ R-\left\{\left(s_{k-1}, s_{k}\right)\right\}, \text { if }\left(s_{k}, s_{k+1}\right) \notin R \text { and }\left(s_{k-1}, s_{k}\right) \in R \\ \left(R-\left\{\left(s_{k-1}, s_{k}\right),\left(s_{k}, s_{k+1}\right\}\right) \cup\left\{\left(s_{k-1}, s_{k+1}\right)\right\}, \text { otherwise }\right.\end{array}\right.$

$L^{\prime}: S^{\prime} \rightarrow 2^{A P}$, where $\forall s \in S^{\prime}$, if $s \in S$, then $L^{\prime}(s)=L(s)$, else

$L^{\prime}\left(s_{\text {new }}\right)=\tau\left(s_{\text {new }}\right)$, where $\tau$ is the truth assignment related to $s_{\text {new }}$.

-State Substitution: $\operatorname{sub}\left(M, s_{\text {new }}, s_{k}\right)=M^{\prime}=\left(S^{\prime}, S^{\prime}{ }_{0}, R^{\prime}, L^{\prime}\right)$ over $A P$, where

$S^{\prime}=\left(S-\left\{s_{k}\right\}\right) \cup\left\{s_{n e w}\right\} ;$

$S^{\prime}{ }_{0}=\left\{\begin{array}{l}\left(S_{0}-\left\{s_{k}\right\}\right) \cup s_{n e w}, \text { if } s_{k} \in S_{0} ; \\ S_{0}, \text { otherwise; }\end{array}\right.$

$R^{\prime}=\left\{\begin{array}{l}\left(R-\left\{\left(s_{k}, s_{k+1}\right)\right\}\right) \cup\left\{\left(s_{\text {new }}, s_{k+1}\right)\right\}, \text { if } s_{k} \in S_{0} \text { and }\left(s_{k}, s_{k+1}\right) \in R ; \\ \left(R-\left\{\left(s_{k-1}, s_{k}\right)\right\}\right) \cup\left\{\left(s_{k-1}, s_{\text {new }}\right)\right\}, \text { if }\left(s_{k}, s_{k+1}\right) \notin R \text { and }\left(s_{k-1}, s_{k}\right) \in R ; \\ R-\left\{\left(s_{k-1}, s_{k}\right),\left(s_{k}, s_{k+1}\right)\right\} \cup\left\{\left(s_{k-1}, s_{\text {new }}\right),\left(s_{\text {new }}, s_{k+1}\right)\right\}, \text { otherwise; }\end{array}\right.$

$L^{\prime}: S^{\prime} \rightarrow 2^{A P}$, where $\forall s \in S^{\prime}$, if $s \in S$, then $L^{\prime}(s)=L(s)$, else

$L^{\prime}\left(s_{\text {new }}\right)=\tau\left(s_{\text {new }}\right)$, where $\tau$ is the truth assignment related to $s_{\text {new }}$.

A simple modification $\delta$ denotes one of the above three single operations. $\delta(M)$ denotes the resulting LTL Kripke model after the simple modification $\delta$ is applied on $M$. For Definition 4, we illustrate "State addition" and "State deletion" operations in detail. "State substitution" is actually the combination of the former two operations and for brevity its illustration is omitted.

In Fig. 2,the original LTL model is $M=\left(S, S_{0}, R, L\right)$, where $S=\left\{s_{0}, s_{1}, s_{2}, s_{3}\right\}$, $S_{0}=\left\{s_{0}\right\}, R=\left\{\left(s_{0}, s_{1}\right),\left(s_{1}, s_{2}\right),\left(s_{2}, s_{3}\right)\right\}$ and $L$ assigns all states $s_{0}, s_{1}, s_{2}$ and $s_{3}$ in $M$ with $\{a\},\{b\},\{c\}$, and $\{d\}$ respectively. We suppose that there are three different simple modifications under the addition operation $\operatorname{add}\left(M, s_{n e w}, s_{k}, s_{k+1}\right)$ as $(\mathrm{A}),(\mathrm{B})$ and $(\mathrm{C})$ in this figure.

In (A), $s_{k}=s_{1}$ and $s_{k+1}=s_{2}$; the modified LTL model is $M^{\prime}=\left(S^{\prime}, S_{0}^{\prime}, R^{\prime}, S^{\prime}\right)$, where $S^{\prime}=\left\{s_{0}, s_{1}, s_{2}, s_{3}, s_{\text {new }}\right\}, S_{0}^{\prime}=\left\{s_{0}\right\}, R^{\prime}=\left\{\left(s_{0}, s_{1}\right),\left(s_{1}, s_{\text {new }}\right),\left(s_{\text {new }}, s_{2}\right)\right.$, $\left.\left(s_{2}, s_{3}\right)\right\}$ and $L^{\prime}$ assigns all states $s_{0}, s_{1}, s_{2}, s_{3}$ and $s_{\text {new }}$ in $M^{\prime}$ with $\{a\},\{b\},\{c\}$, $\{d\}$ and $\{$ new $\}$ respectively.

In (B), $s_{k}=\emptyset$ and $s_{k+1}=s_{0}$; the modified LTL model is $M^{\prime}=\left(S^{\prime}, S_{0}^{\prime}, R^{\prime}, S^{\prime}\right)$, where $S^{\prime}=\left\{s_{0}, s_{1}, s_{2}, s_{3}, s_{\text {new }}\right\}, S_{0}^{\prime}=\left\{s_{\text {new }}\right\}, R^{\prime}=\left\{\left(s_{\text {new }}, s_{0}\right),\left(s_{0}, s_{1}\right),\left(s_{1}, s_{2}\right)\right.$, $\left.\left(s_{2}, s_{3}\right)\right\}$ and $L^{\prime}$ assigns all states $s_{0}, s_{1}, s_{2}, s_{3}$ and $s_{\text {new }}$ in $M^{\prime}$ with $\{a\},\{b\},\{c\}$, $\{d\}$ and $\{$ new $\}$ respectively.

In (C), $s_{k}=s_{3}$ and $s_{k+1}=\emptyset$; the modified LTL model is $M^{\prime}=\left(S^{\prime}, S_{0}^{\prime}, R^{\prime}, S^{\prime}\right)$, where $S^{\prime}=\left\{s_{0}, s_{1}, s_{2}, s_{3}, s_{\text {new }}\right\}, S_{0}^{\prime}=\left\{s_{0}\right\}, R^{\prime}=\left\{\left(s_{0}, s_{1}\right),\left(s_{1}, s_{2}\right),\left(s_{2}, s_{3}\right)\right.$,

$\left.\left(s_{3}, s_{\text {new }}\right)\right\}$ and $L^{\prime}$ assigns all states $s_{0}, s_{1}, s_{2}, s_{3}$ and $s_{\text {new }}$ in $M^{\prime}$ with $\{a\}$, $\{b\},\{c\},\{d\}$ and $\{$ new $\}$ respectively.

In Fig. 3, the deletion operation delete $\left(M, s_{k}\right)$ works on the same LTL model as above. (A), (B) and (C) in this figure are three different simple modifications from the deletion operation with $s_{k}=s_{2}, s_{k}=s_{0}$, and $s_{k}=s_{3}$ respectively. 


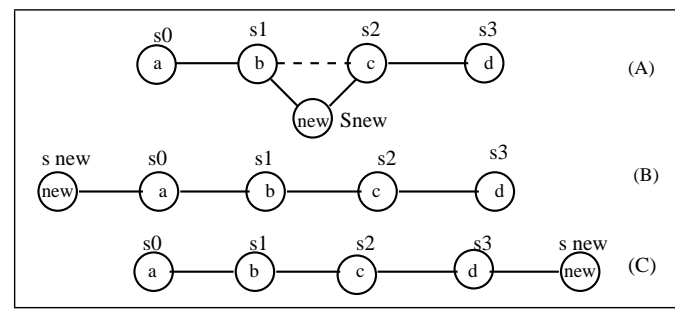

Fig. 2. The illustration of the addition operation.

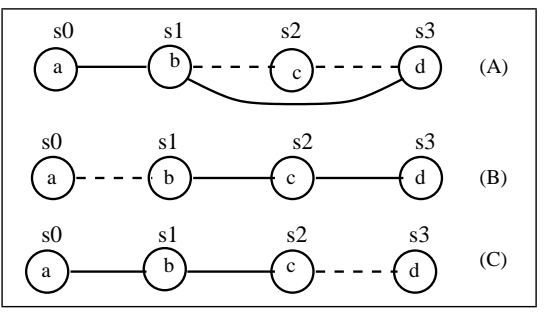

Fig. 3. The illustration of the deletion operation.

The modified LTL model in (A) is $M^{\prime}=\left(S^{\prime}, S_{0}^{\prime}, R^{\prime}, S^{\prime}\right)$, where $S^{\prime}=\left\{s_{0}, s_{1}, s_{3}\right\}$, $S_{0}^{\prime}=\left\{s_{0}\right\}, R^{\prime}=\left\{\left(s_{0}, s_{1}\right),\left(s_{1}, s_{3}\right)\right\}$ and $L^{\prime}$ assigns all states $s_{0}, s_{1}, s_{3}$ of $M^{\prime}$ with $\{a\},\{b\}$ and $\{d\}$ respectively.

The modified LTL model in (B) is $M^{\prime}=\left(S^{\prime}, S_{0}^{\prime}, R^{\prime}, S^{\prime}\right)$, where $S^{\prime}=\left\{s_{1}, s_{2}, s_{3}\right\}$, $S_{0}^{\prime}=\left\{s_{1}\right\}, R^{\prime}=\left\{\left(s_{1}, s_{2}\right),\left(s_{2}, s_{3}\right)\right\}$ and $L^{\prime}$ assigns all states $s_{1}, s_{2}$, and $s_{3}$ of $M^{\prime}$ with $\{b\},\{c\},\{d\}$ respectively.

The modified LTL model in (C) is $M^{\prime}=\left(S^{\prime}, S_{0}^{\prime}, R^{\prime}, S^{\prime}\right)$, where $S^{\prime}=\left\{s_{0}, s_{1}, s_{2}\right\}$, $S_{0}^{\prime}=\left\{s_{0}\right\}, R^{\prime}=\left\{\left(s_{0}, s_{1}\right),\left(s_{1}, s_{2}\right)\right\}$ and $L^{\prime}$ assigns all states $s_{0}, s_{1}$ and $s_{2}$ of $M^{\prime}$ with $\{a\},\{b\}$, and $\{c\}$ respectively.

Definition 5. (Update) Given an LTL Kripke model $M$ and an LTL formula $\phi$. An update of $M$ with $\phi$, denoted as $\operatorname{Update}(M, \phi)$, is a new LTL Kripke model $M^{\prime}$, such that

1. there exists a sequence of simple modifications $\left(\delta_{0}, \delta_{1}, \delta_{2}, \cdots, \delta_{n}\right)$ for $\left(M_{0}, M_{1}, M_{2}, \cdots, M_{n-1}, M_{n}\right)$ respectively, where $M_{0}=M$. The simple modifications are $\delta_{0}\left(M_{0}\right)=M_{1}, \delta_{1}\left(M_{1}\right)=M_{2}, \cdots, \delta_{i}\left(M_{i}\right)=M_{i+1}, \cdots$, and $\delta_{n}\left(M_{n}\right)=$ $M^{\prime}$ respectively, where $0 \leq i \leq n$. $M^{\prime}$ is the result of the $(n+1)^{t h}$ simple modification;

2. $M^{\prime} \models \phi$.

In Definition 5 , the update $U p d a t e(M, \phi)$ will result in a new LTL model $M^{\prime}$, which satisfies $\phi$. The method of update is to apply any one of the three single operations in Definition $4 n+1$ times, until $M^{\prime} \models \phi$. The process is viewed as the sequence: $M_{0} \stackrel{\delta_{0}}{\longrightarrow} M_{1} \stackrel{\delta_{1}}{\longrightarrow} M_{2}, \cdots, M_{n} \stackrel{\delta_{n}}{\longrightarrow} M_{n+1}$, where we suppose the first simple modification $\delta_{0}$ by using a single operation, the second one $\delta_{1}$, and so on, the last one $\delta_{n}$. Consequently, the first simple modification works on $M$, denoted as $\delta_{0}(M)=M_{1}$; the second one $\delta_{1}$ works on $M_{1}$, denoted as $\delta_{1}\left(M_{1}\right)=M_{2}$ and so on; the last one $\delta_{n}$ works on $M_{n}$, denoted as $\delta_{n}\left(M_{n}\right)=M^{\prime}$, which satisfies $\phi$. In other words, $\operatorname{Update}(M, \phi)$ consists of the sequence of simple modifications $\delta_{0}, \delta_{1}, \cdots, \delta_{n}$, and gives a final result $M^{\prime}$.

\section{The Principles of Minimal Change}

In order to eliminate possible undesirable update results from the modifications in the previous section, we need to provide justified semantic criterion on LTL 
model updating. Basically, we prefer to select only those new models that have least change compared with the original models. In belief revision and update research, various minimal change principles have been developed based on classical propositional logic such as described in [12]. It has been observed that these principles are usually not directly applicable for updating on modal logic based systems such as those described in [1], which works on the $S 5$ modal logic Kripke model. In this section, we propose a new minimal change definition for the LTL Kripke model update, based on both logic structure comparison and state differences of LTL Kripke models.

Given a set $X,|X|$ denotes the cardinality of $X$. Given an integer, $N$, which could be either positive or negative. $\|N\|$ denotes its absolute value. e.g., $\|-3\|=$ $\|3\|=3$. Given any two sets $X$ and $Y$, the symmetric difference between $X$ and $Y$ is denoted as $\operatorname{Diff}(X, Y)=(X-Y) \cup(Y-X)$.

\section{Definition 6. (Ordering on Relations)}

Given three LTL models $M=\left(S, S_{0}, R, L\right), M_{1}=\left(S_{1}, S_{1_{0}}, R_{1}, L_{1}\right)$ and $M_{2}=$ $\left(S_{2}, S_{2_{0}}, R_{2}, L_{2}\right)$, we say relation $R_{1}$ in $M_{1}$ is as close to relation $R$ in $M$ as relation $R_{2}$ in $M_{2}$, denoted as $R_{1} \leq_{R} R_{2}$, if

1. $\forall\left(s_{i}, s_{i+1}\right) \in\left(R-R_{1}\right),\left(s_{i}, s_{i+1}\right) \in\left(R-R_{2}\right)$, and

2. $\left\|\left(|R|-\left|R_{1}\right|\right)|| \leq\right\|\left(|R|-\left|R_{2}\right|\right) \|$.

Furthermore, $R_{1}=_{R} R_{2}$ iff $R_{1} \leq_{R} R_{2}$ and $R_{2} \leq_{R} R_{1} ; R_{1}<_{R} R_{2}$ if $R_{1} \leq_{R} R_{2}$ and $R_{1} \neq{ }_{R} R_{2}$.

In Definition 6, condition 1 means that any element of $R$ that is not retained in $R_{1}$ is also not retained in $R_{2}$; condition 2 indicates that the different number of elements between $R$ and $R_{1}$ is less than or at most the same as those between $R$ and $R_{2}$.

To illustrate how this definition works, let us consider Figure 4 . It is easy to see that $R-R_{1}=\left\{\left(s_{2}, s_{3}\right),\left(s_{3}, s_{4}\right)\right\}$ and $\left.R-R_{2}=\left\{\left(S_{1}, S_{2}\right)\right),\left(S_{2}, S_{3}\right),\left(S_{3}, S_{4}\right)\right\}$. Thus $R-R_{1} \subset R-R_{2}$, and condition 1 in Definition 6 is satisfied. Also we have $\left\|\left(|R|-\left|R_{1}\right|\right)\right\|=|| 4-3 \|=1$, and $\left\|\left(|R|-\left|R_{2}\right|\right)||=\right\| 4-2||=2$. That is $\left\|\left(|R|-\left|R_{1}\right|\right)||<\right\|\left(|R|-\left|R_{2}\right|\right) \|$, so condition 2 is also satisfied. Therefore, we have $R_{1} \leq_{R} R_{2}$. In other words, in terms of relations, model $M_{1}$ is closer to $M$ than model $M_{2}$.

Definition 7. (Ordering on Models) Giving $M, M_{1}$, and $M_{2}$, we say $M_{1}$ is as close to $M$ as $M_{2}$, which is denoted as $M_{1} \leq_{M} M_{2}$, if the following conditions hold:

1. $R_{1}<{ }_{R} R_{2}$, or

2. if $R_{1}={ }_{R} R_{2}$, then, for $\forall\left(s_{i}, s_{i+1}\right) \in R, \exists\left(s_{i}^{\prime}, s_{i+1}^{\prime}\right) \in R_{1}$, such that, $\forall\left(s_{i}{ }_{i}, s_{i+1}^{\prime \prime}\right) \in R_{2}, \operatorname{Diff}\left(s_{i}, s_{i}^{\prime}\right) \subseteq \operatorname{Diff}\left(s_{i}, s_{i}^{\prime \prime}\right)$ implies

$\operatorname{Diff}\left(s_{i+1}, s_{i+1}^{\prime}\right) \subseteq \operatorname{Diff}\left(s_{i}, s_{i+1}^{\prime \prime}\right)$.

We denote $M_{1}<_{M} M_{2}$ iff $M_{1} \leq_{M} M_{2}$ and $M_{2} \mathbb{L}_{M} M_{1}$. 


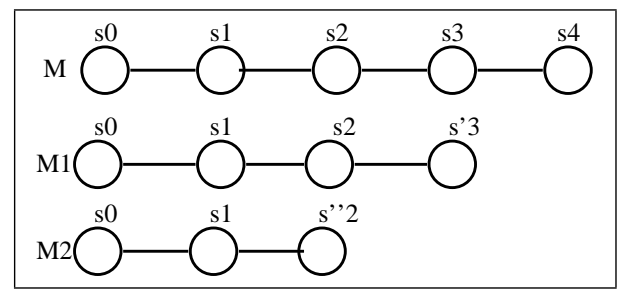

Fig. 4. The illustration of Definition 6 .

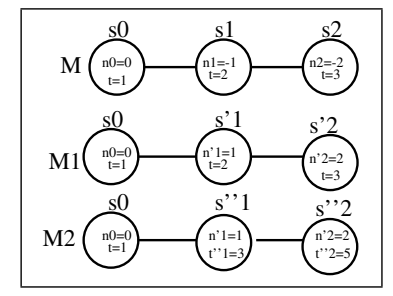

Fig. 5. Illustrate minimal changed update for example 1 and Definition 7.

Definition 8. (Admissible Update) Given an LTL Kripke model $M$ and an $L T L$ formula $\phi$, an update of $M$ with $\phi$, denoted as $\operatorname{Update}(M, \phi)$, is called admissible, if any resulting model $M^{\prime}$, obtained from $\operatorname{Update}(M, \phi)$, has the following properties:

1. $M^{\prime} \models \phi$;

2. There does not exist a resulting model $M^{\prime \prime}$ from other update of $M$ with $\phi$, such that $M^{\prime \prime} \models \phi$ and $M^{\prime \prime}<_{M} M^{\prime}$.

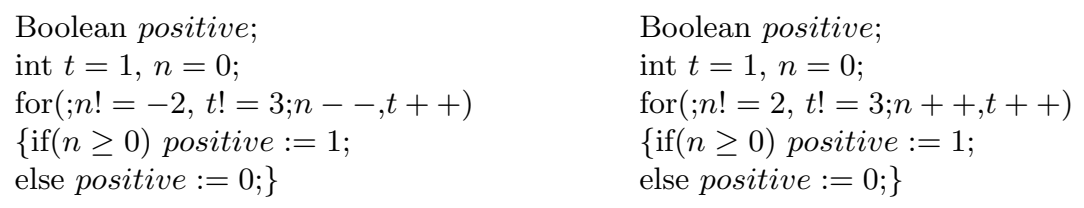

Fig. 6. The original code for example 1. Fig. 7. The updated code for example 1.

Example 1. Consider the software code illustrated in Figure 6. We need the software system $M$ to satisfy the property: $M=G$ (positive), which means "positive $=$ True" when all states of path $\pi$ in $M$ contain "n=positive number". We denote $\phi=G$ (positive).

Now, we have model $M=\left(S, S_{0}, R, L\right)$ in Figure 5 to represent the code in Figure 6, and $M \not \forall \phi$. We give up most other obvious none minimal changed updated models and pick up two updated models $M_{1} \models \phi$ and $M_{2} \models \phi$ in the figure for comparison. To result in both new models, the operation $\operatorname{sub}\left(M, S_{n e w}, s_{k}\right)$,i.e., the simple modification should be applied twice in each. Now we shall pick out a minimal changed model between $M_{1}$ and $M_{2}$.

Under Definition 6 , we have $R_{1}={ }_{R} R_{2}$. We need to apply Definition 7 to decide the desirable update. Under condition 2 of this definition, Diff $\left(s_{1}, s_{1}^{\prime}\right)=$ $\left\{n_{1}=-1, n_{1}^{\prime}=1,\right\}$ and $\operatorname{Diff}\left(s_{1}, s_{1}^{\prime \prime}\right)=\left(n_{1}=-1, n_{1}^{\prime}=1, t=2, t=3\right)$ resulting in $\operatorname{Diff}\left(s_{1}, s_{1}^{\prime}\right) \subset \operatorname{Diff}\left(s_{1}, s_{1}^{\prime \prime}\right)$. Similarly, it is easy to see $\operatorname{Diff}\left(s_{2}, s_{2}^{\prime}\right) \subset$ $\operatorname{Diff}\left(s_{2}, s_{2}^{\prime \prime}\right)$.

Thus, from the above comparison, $M_{1}<_{M} M_{2}$, we have final admissible minimal changed model $M_{1} \models \phi$, which leads to the final updated software code in Fig. 7 . 


\section{Characterization}

In this section, we will characterize the update of the LTL Kripke model with $F \phi$ and $\phi \cup \psi$, which are two of the most common and frequently used path formulae in LTL model checking. For simplicity, we consider those LTL Kripke models that only contain a single initial state. However, our results can easily be extended to the general case. The characterizations significantly simplify the underlying update process for these specific formulas.

Theorem 1. Given $M=\left(S,\left\{s_{0},\right\}, R, L\right)$ with a path $\pi=\left[s_{0}, \cdots, s_{k-1}, s_{k}, s_{k+1}\right.$, ...], where $s_{k+1}$ is not the final state, and $M \forall F F$. Update $(M, F \phi)=M^{\prime}$, where $M^{\prime} \models F \phi$ and $M^{\prime}=\left(S^{\prime},\left\{s_{0}\right\}, R^{\prime}, L^{\prime}\right)$, is admissible if the update generates one of the following resulting models by applying the simple modifications:

1. Addition operation add $\left(M, s_{\text {new }}, s_{k}, s_{k+1}\right)$. The updated model $M^{\prime}$ has a path $\pi^{\prime}=\left[s_{0}, \cdots, s_{k}, s_{\text {new }}, s_{k+1}, \cdots\right]$, such that,

$-S^{\prime}=S \cup\left\{s_{\text {new }}\right\}, R^{\prime}=R-\left\{\left(s_{k}, s_{k+1}\right)\right\} \cup\left\{\left(s_{k}, s_{\text {new }}\right),\left(s_{\text {new }}, s_{k+1}\right)\right\}$, and

$L^{\prime}: S^{\prime} \rightarrow 2^{A P}$, where $\forall s \in S^{\prime}$, if $s \in S$, then $L^{\prime}(s)=L(s)$;

-Diff $\left(S_{k+1}, s_{\text {new }}\right)$ is minimal and $s_{\text {new }}=\phi$.

2. Substitution operation $\operatorname{sub}\left(M, s_{\text {new }}, s_{k}\right)$. The updated model $M^{\prime}$ has a path $\pi^{\prime}=\left[s_{0}, \cdots, s_{k-1}, s_{n e w}, s_{k+1}, \cdots\right]$, such that,

$-S^{\prime}=S-\left\{s_{k}\right\} \cup\left\{s_{\text {new }}\right\}$,

$R^{\prime}=R-\left\{\left(s_{k-1}, s_{k}\right),\left(s_{k}, s_{k+1}\right)\right\} \cup\left\{\left(s_{k-1}, s_{\text {new }}\right),\left(s_{\text {new }}, s_{k+1}\right)\right\}$, and

$L^{\prime}: S^{\prime} \rightarrow 2^{A P}$, where $\forall s \in S^{\prime}$, if $s \in S$, then $L^{\prime}(s)=L(s)$;

-Diff $\left(S_{k}, s_{\text {new }}\right)$ is minimal and $s_{\text {new }}=\phi$.

Theorem 2. Given $M=\left(S,\left\{s_{0},\right\}, R, L\right)$ with a path $\pi=\left[s_{0}, \cdots, s_{k}, \cdots, s_{j}, \cdots\right]$, where $M \not \models \phi \cup \psi$. An update $\operatorname{Update}(M, \phi \cup \psi)=M^{\prime}$, where $M^{\prime} \models \phi \cup \psi$, is admissible by applying the simple modification: substitution $\operatorname{sub}\left(M, s_{k}^{\prime}, s_{k}\right)$ to any state $s_{k}$ before $s_{j}$ in $M$, where $s_{k} \not \models \phi, s_{j}$ is the first state with $s_{j} \models \psi$ in $\pi$, and Diff $\left(s_{k}, s_{k}^{\prime}\right)$ is minimal. The resulting model $M^{\prime}=\left(S^{\prime},\left\{s_{0}^{\prime}\right\}, R^{\prime}, L^{\prime}\right)$ with $\pi^{\prime}=\left[s_{0}^{\prime}, \cdots, s_{k}^{\prime}, \cdots, s_{j}, \cdots\right]$ such that,

$-S^{\prime}=S-\left\{s_{k} \mid k<j, s_{k} \not \models \phi\right\} \cup\left\{s_{k}^{\prime} \mid k<j, s_{k}^{\prime}=\phi\right\}$,

$-s_{0}^{\prime}=s_{0}$ if $s_{0} \models \phi$, otherwise, $s_{0}^{\prime}=\phi$ and Diff $\left(s_{0}^{\prime}, s_{0}\right)$ is minimal,

$-R^{\prime}=R-\left\{\left(s_{k}, s_{k+1}\right) \mid k \leq(j-1), s_{k} \not \models \phi\right.$ or

$\left.s_{k+1} \not \models \phi\right\} \cup\left\{\left(s_{k}^{\prime}, s_{k+1}^{\prime}\right) \mid k \leq(j-1)\right.$, and $\left.\left(s_{k}^{\prime}, s_{k+1}^{\prime}\right) \subseteq \pi^{\prime}\right\}$, and

$-L^{\prime}: S^{\prime} \rightarrow 2^{A P}$, where $\forall s \in S^{\prime}$, if $s \in S$, then $L^{\prime}(s)=L(s)$.

\section{Conclusions, Related Work and Further Research}

In this paper, we have described a new approach of system modification for LTL representation. The LTL model updater achieves its modification task by three single operations. A principle of minimal change is also presented for our LTL model update approach. We further demonstrate examples and characterize two important properties of the LTL model to illustrate the update and minimal change criterion. 
Our work presented in this paper can be viewed as a further development of enhanced model checking for system repair by AI techniques, as shown in [2], which introduced the concept of a system repair problem. The authors in that paper integrated the techniques from model checking and abductive AI principles to tackle automated diagnosis. However, as declared by the authors themselves, their approach may not be general enough for other system modification problems. The other related work is due to Harris and Ryan's work [5]. In that paper, model checking is formalized as belief updating operator $\diamond$ to satisfy classical proposition knowledge update KM postulates $U 1-U 8$. However, it is not clear how their model update operator can be implemented for practical system modification.

In order to further develop our research, we will aim to perform an extensive case study of the LTL model update to provide strong supportive evidence for the general principles proposed in this paper. We will apply the LTL model update method to more complex software systems such as concurrent systems [2]. We will also consider alternative methods to formalize the minimal change criterion to enlarge the LTL model update application domain. After this stage of research, we will extend our current approach to the research of CTL model update, which will be more of a challenge and the main focus of our research.

\section{References}

1. Baral, C. and Zhang, Y. (2005). Knowledge updates: Semantics and complexity issues. To appear Artificial Intelligence (AIJ), 2005.

2. Buccafurri, F. et al. (1999). Enhancing model checking in verification by AI techniques. Artificial Intelligence 112(1999) 57-104.

3. Clarke, E. Jr., Grumberg, O. and Peled, D. (1999). Model Checking. The MIT Press, Cambridge, Massachusetts London, England.

4. Gammie, P. and van der Meyden, R.(2004). MCK-Model checking the logic of knowledge. In proceeding of 16 th International conference on Computer Aided Verification.

5. Harris,H. and Ryan,M.(2003). Theoretical foundations of updating systems. In the prodeeding of the 18th IEEE International Conference on Automated Software Engineering.

6. Herzig, A. and Rifi, O. (1999). Propositional belief base update and minimal change. Artificial Intelligence 115(1999)107-138. Elsevier Science.

7. Holzmann, G. (2003). The SPIN Model Checker: Primer and Reference Manual. Addison-Wesley Professional.

8. Huth, M. and Ryan, M. (2000). Logic in Computer Science: Modelling and Reasoning about Systems. University Press, Canbridge.

9. McMillan,K. and Amla,N.(2002). Automatic abstraction without counterexamples. Cadence Berkeley Labs, Cadence Design Systems.

10. Stumptner,M. and Wotawa,F.(1998). A survey of intelligent debugging. AI Communications, 11(1),1998.

11. Vaziri-Farahani, M. (1995). Using symbolic model checking to verify cache coherence in a distributed file system. Tech. Rep. CMU-CS-95-156, Carnegie Mellon Computer Science Department. Bachelor's Thesis.

12. Winslett, M. (1990). Updating logical databases. Cambridge University Press. 